\title{
Dehydrozingerone analogues in synthesis attractive sulfonamide compounds as potential antitumor agents
}

\author{
Zoran Ratković ${ }^{1}$, Suzana Popović ${ }^{3}$, Sanja Matić ${ }^{4}$, Danijela Todorović ${ }^{5}$, Dejan Baskić ${ }^{3}$ and Jovana \\ Muškinja1,2,* \\ 1 Department of Chemistry, Faculty of Science, University of Kragujevac, Radoja Domanovića 12, \\ 34000 Kragujevac; wor@kg.ac.rs \\ 2 Department of Sciences, Institute for Information Technologies, University of Kragujevac, Jovana Cvijića bb, \\ 34000 Kragujevac, Serbia; \\ 3 Department of Microbiology and Immunology, Faculty of Medical Sciences, University of Kragujevac, \\ Svetozara Markovića 67, 34000 Kragujevac, Serbia; popovic007@yahoo.com, dejan.baskic@gmail.com \\ 4 Department of Pharmaceutical Technology, Faculty of Medical Sciences, University of Kragujevac, \\ Svetozara Markovića 67, Serbia; sanjad.matic@gmail.com \\ 5 Department of Genetics, Faculty of Medical Sciences, University of Kragujevac, Svetozara Markovića 67, \\ Serbia; dtodorovic197@gmail.com \\ *Correspondence: jovana.muskinja@gmail.com;
}

\begin{abstract}
Sulfonamides are very important scaffold in design of different compounds with potential biological activity. They are the basis of several groups of drugs and could be called sulfa drugs. Starting from this fact, we wanted to synthesize some new types of sulfonamides, prepared from derivatives of natural product vanillin. In light of this, we synthesized dehydrozingerone, 4-(4-hydroxy-3-methoxyphenyl)-3-buten-2-one, and its alkyl derivatives. These compounds served as good substrates in the synthesis of new sulfonamide compounds. The structures of the new compounds were determined by IR and NMR methods. Cytotoxicity screening of fourteen new organic compounds against SW480, Hela and MRC-5 was measured by colorimetric MTT assay after $48 \mathrm{~h}$ of treatment. Cytotoxicity ratio (CR) was calculated as \% of cytotoxicity of each compound on normal cells and \% of cytotoxicity of the same compounds on tumor cell line. CR is pointed to some new compounds as promising candidates for further experiments.
\end{abstract}

Keywords: sulfonamide; cytotoxic activity; enone system; chalcones; pyrazoline

\section{Introduction}

Natural products serve as a key source for the design, discovery and development of potentially novel drugs. Ginger root is excellent source for many kinds of active compounds and from this plant various kinds of compounds were isolated. One of main constituent is dehydrozingerone (Figure 1). He exhibits a wide range of biological activity [1-3] and could be easily transformed.<smiles>COc1cc(/C=C/C(C)=O)ccc1O</smiles>

Figure 1. Structure of dehydrozingerone 
Some of very interesting and useful reactions for these types of compounds, which have enone system in their structure, are transformation into heterocyclic derivatives, in reactions with urea, thiourea, hydroxylamine, hydrazine, guanidine [4-6]. By this way various usable heterocyclic derivatives, such as pyrimidines, pyrazolines, pyrazoles, oxazoles, thiazoles... were obtained. The pyrazolines are the five membered heterocyclic compounds and extensive important synthons which have attracted the attentions of organic chemists in the past decades due to their immense biological applications. These kind of compounds have played an important role in the development of theoretical heterocyclic chemistry and some of their various spectra of biological activities are antiinflammatory, antidepressant, antibacterial, antiviral, anticancer activities [7-9] and antidiabetic activities [10].

On the other hand, sulfonamide derivatives represents another important class of organic compounds that displayed interesting biological activities [11,12] as the sulfonamide group is an important structural core in medicinal chemistry. These compounds are the first successfully synthesized selectively toxic antimicrobial drugs [13]. Predominantly, in last decades a large number of structurally novel sulfonamide derivatives have been described as potential anticancer reagents [11,14]. All these derivatives incorporate in their molecules a common chemical motif of aromatic/heterocyclic sulfonamides. Some of them is Indisulam (Figure 2) (E7070; $\mathrm{N}$-(3-chloro-7-indolyl)-1,4-benzenedisulfonamide), a novel sulfonamide anticancer agent in clinical development for the treatment of solid tumors [15].

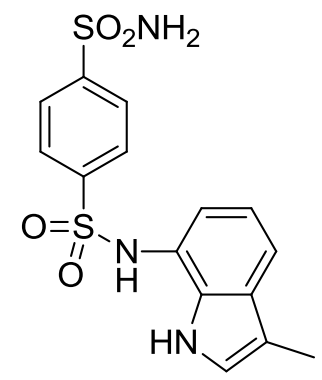

Figure 2. Structure of indisulam (E7070)

Starting from this fact and from our previous results in dehydrozingerone derivatives transformation [16], we expected that incorporation of different pharmacophore into the same molecule might have an attracting structural result for development of novel antitumor agents. In the present work, we described the synthesis of novel compounds having vanillin core, pyrazoline fragment and sulfonamide group in their structure, under assumption that they could have potentially promising activity.

\section{Results and Discussion}

\subsection{Chemistry synthesis}

In a reaction Claisen-Schmidt condensation of vanillin and acetone, dehydrozingerone 1, were prepared. Keeping in mind the reactivity of phenolic functional group, the synthesis of $O$-alkyl dehydrozingerone derivatives was carried out as a simple reaction free phenolic group in the boiling acetone solution in the presence of corresponding alkyl halide. Two alkyl derivatives, 4-(3-methoxy-4-propoxyphenyl)-3-buten-2-ones (2a) and 4-(4-butoxy-3-methoxyphenyl)-3-buten-2-ones (2b), were obtained, according to the described literature procedures [17] (Scheme 1). 


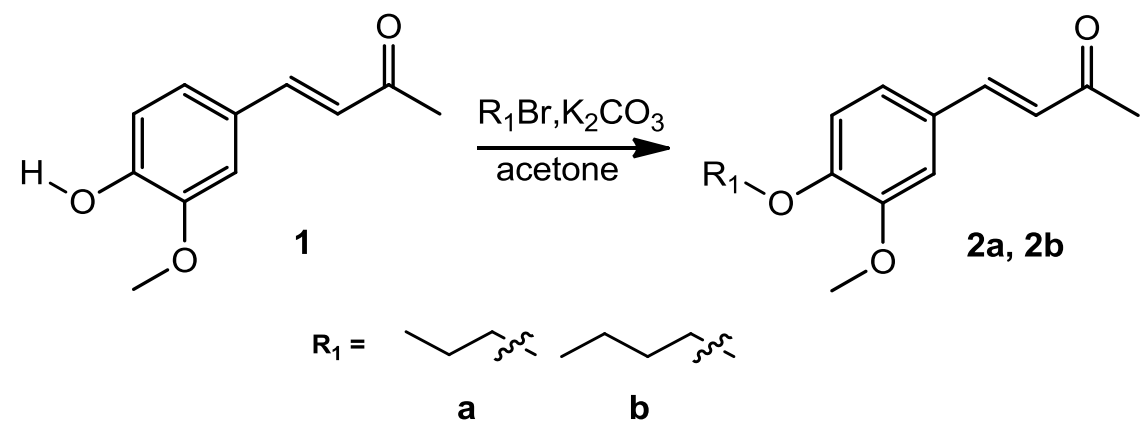

Scheme 1. Synthesis of $O$-alkyl derivatives of dehydrozingerone

Enone compounds $\mathbf{2 a}$ and $\mathbf{2 b}$ react with hydrazine hydrochloride and pyrazoline derivatives were synthesized. The $\mathrm{N}-\mathrm{H}$ group in pyrazoline compounds is very reactive and a good target for the attack of different reagents. Further reaction was carried in presence of various sulfonyl chlorides and trimethylamine, yielding a new sulfonamide compounds $3\left(a^{\prime}-g^{\prime}\right)$ and $\mathbf{4}\left(\mathbf{a}^{\prime}-\mathrm{g}^{\prime}\right)$ (Scheme 2).

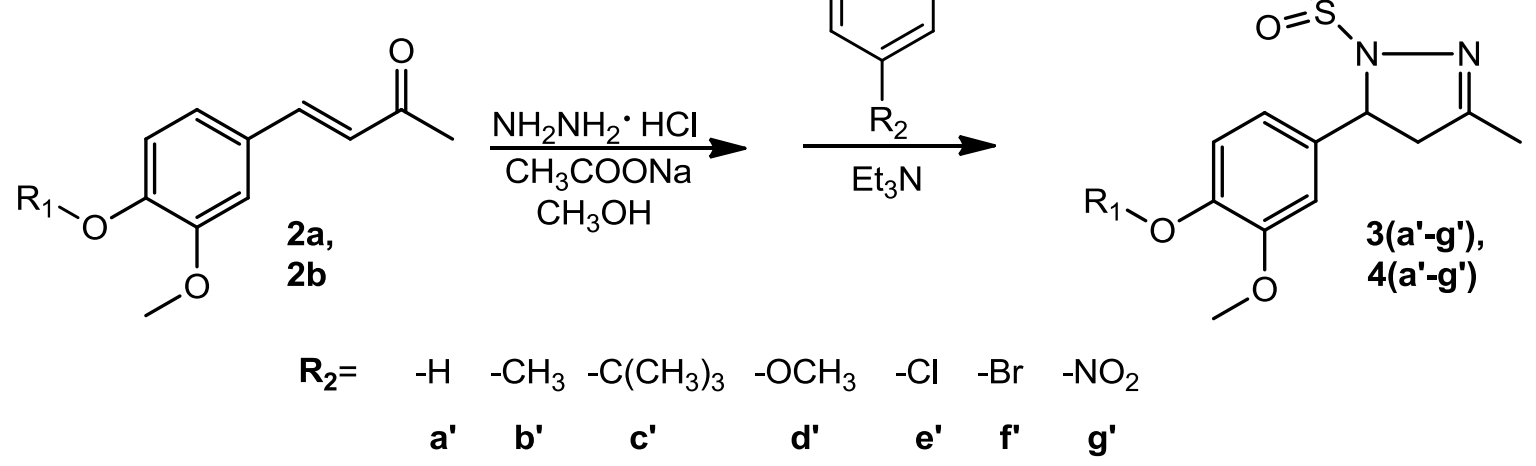

Scheme 2. Synthesis of novel sulfonamide compounds

All new compounds were well characterized by $\mathrm{IR},{ }^{1} \mathrm{H},{ }^{13} \mathrm{C}$ NMR and physical data. The structures of all synthesized products $3\left(\mathbf{a}^{\prime}-\mathbf{g}^{\prime}\right)$ and $\mathbf{4}\left(\mathbf{a}^{\prime}-\mathbf{g}^{\prime}\right)$ are presented in Table 1.

Table 1. Synthesized sulfonamide derivatives

Entry Substrate Product


Proceedings 2019, 3, x FOR PEER REVIEW

4 of 11

1<smiles>O=S(=O)(Cl)c1ccccc1</smiles>

2

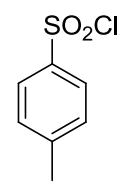

3

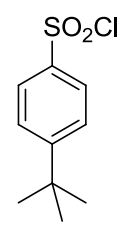

4<smiles>COc1ccc(S(=O)(=O)Cl)cc1</smiles>

5<smiles>O=S(=O)(Cl)c1ccc(Cl)cc1</smiles>

6<smiles>O=S(=O)(Cl)c1ccc(Br)cc1</smiles>

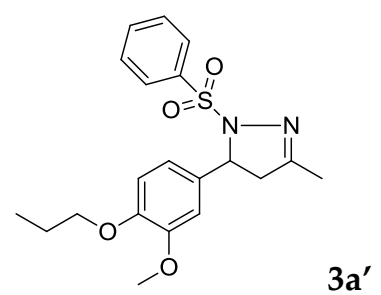

69

88

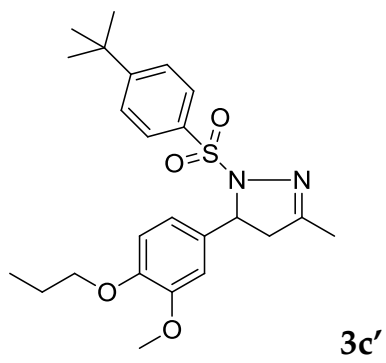

$3 d^{\prime}$

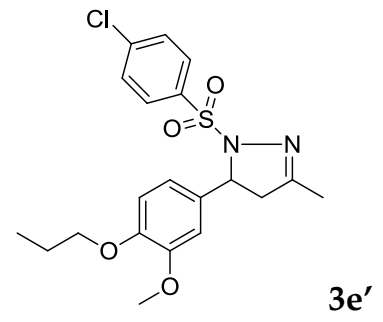

78

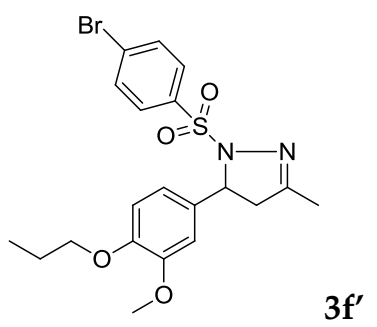

87

85

80 
Proceedings 2019, 3, x FOR PEER REVIEW

5 of 11

7

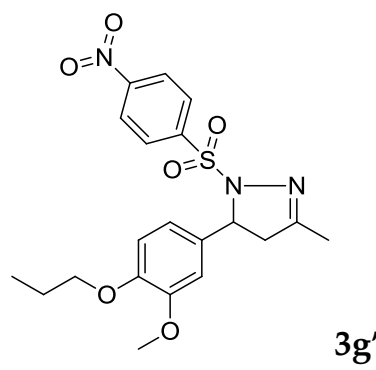

82

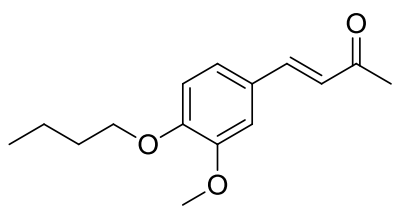

8<smiles>O=S(=O)(Cl)c1ccccc1</smiles>

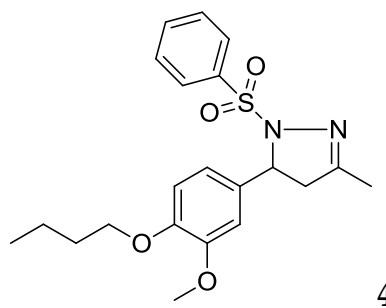

$4 a^{\prime}$

9
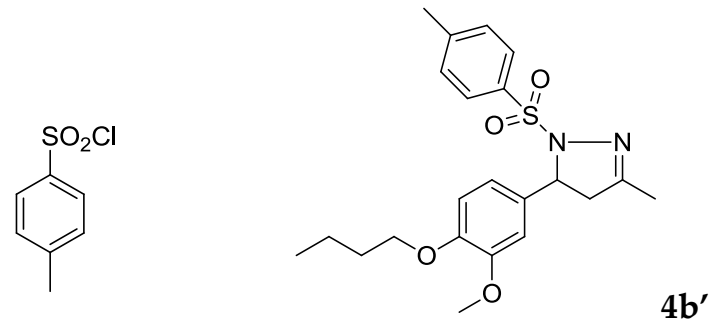

70

90

10
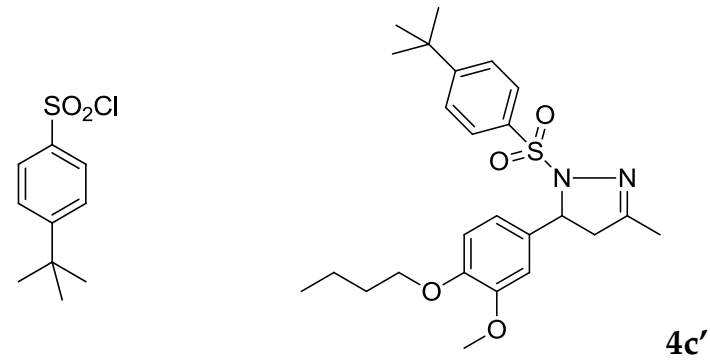

75

11<smiles>COc1ccc(S(=O)(=O)Cl)cc1</smiles>

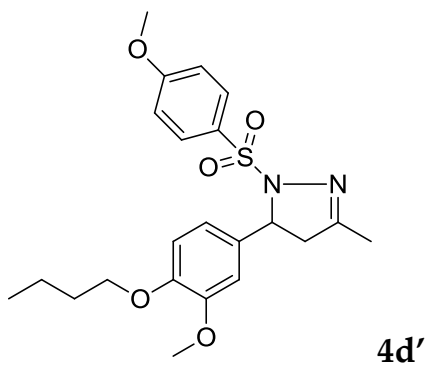

85 
12<smiles>O=S(=O)(Cl)c1ccc(Cl)cc1</smiles>

13<smiles>O=S(=O)(Cl)c1ccc(Br)cc1</smiles>

14<smiles>O=[N+]([O-])c1ccc(S(=O)(=O)Cl)cc1</smiles><smiles>CCCCOc1ccc(C2CC(C)=NN2S(=O)(=O)c2ccc(Cl)cc2)cc1OC</smiles>

\subsection{Cytotoxicity}

Results of MTT assay showing cytotoxicity of fourteen organic compounds on SW480, Hela and MRC-5 cell lines are presented as clustered column chart (Figure 3). CR values of tested compounds for both tumor cell lines are presented in Table 2.

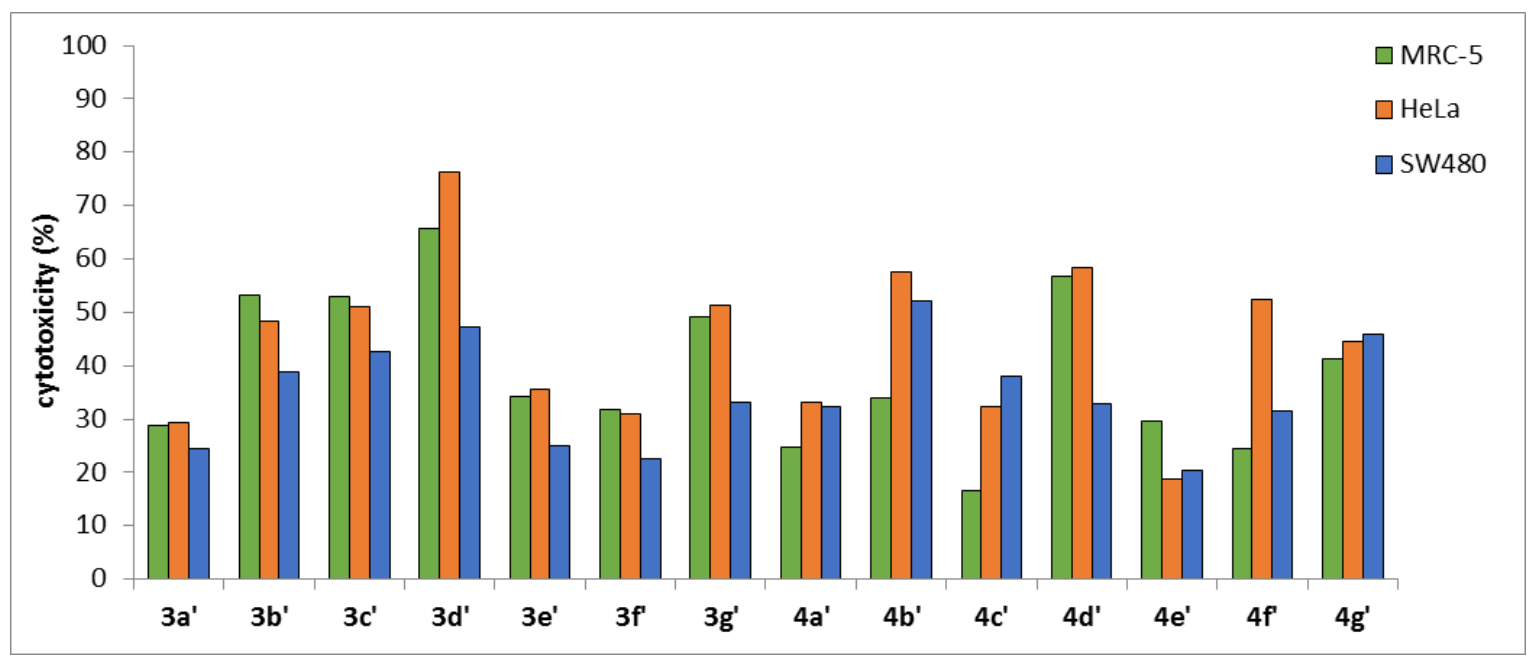

Figure 3. Cytotoxicity of tested organic compounds $(100 \mu \mathrm{M})$ on SW480, Hela and MRC-5 cell lines after $48 \mathrm{~h}$ of treatment

Compounds $3 \mathbf{c}^{\prime}, \mathbf{3} \mathrm{g}^{\prime}, \mathbf{4} \mathbf{b}^{\prime}, \mathbf{4} \mathbf{d}^{\prime}$ and $\mathbf{4} \mathbf{f}^{\prime}$ exerted moderate cytotoxic effect on Hela cells, while compound $\mathbf{3} \mathbf{d}^{\prime}$ showed strong cytotoxicity $(76.19 \%)$. On SW480 cell line compound $\mathbf{4} \mathbf{b}^{\prime}$ exhibited moderate cytotoxic effect. The cytotoxicity ratio (CR), as relative indicator of selective nature of 
compound for tumor cells over non-transformed cells, showed moderate selectivity of compound $\mathbf{4} \mathbf{b}^{\prime}$ for both tumor cell lines, Hela and SW480, while compound $4 \mathbf{f}^{\prime}$ was selective only for Hela cells.

On the other hand, compounds $\mathbf{4} \mathbf{a}^{\prime}$ and $\mathbf{4} \mathbf{c}^{\prime}$ also showed a good ratio, but cytotoxicity on malignant cells at tested concentration was very low (30-40\%), even though they had low effect on healthy cells (MRC-5). Therefore, the best candidates for further testing are compounds $\mathbf{4} \mathbf{b}^{\prime}$ and $\mathbf{4} \mathbf{f}^{\prime}$ on Hela cells, and compound $4 \mathbf{b}^{\prime}$ on SW480 cell line.

Table 2. The cytotoxicity ratio (CR) of tested compounds expressed as quotient of cytotoxicity $\%$ on normal cells and cytotoxicity \% on tumor cell line.

\begin{tabular}{ccc}
\hline & MRC-5/HeLa & MRC/SW480 \\
\hline $\mathbf{3 \mathbf { a } ^ { \prime }}$ & 0.99 & 1.18 \\
$\mathbf{3 \mathbf { b } ^ { \prime }}$ & 1.10 & 1.37 \\
$\mathbf{3 \mathbf { c } ^ { \prime }}$ & 1.04 & 1.24 \\
$\mathbf{3 \mathbf { d } ^ { \prime }}$ & 0.86 & 1.39 \\
$\mathbf{3 \mathbf { e } ^ { \prime }}$ & 0.96 & 1.37 \\
$\mathbf{3 \mathbf { f } ^ { \prime }}$ & 1.02 & 1.41 \\
$\mathbf{3 \mathbf { g } ^ { \prime }}$ & 0.96 & 1.49 \\
$\mathbf{4 \mathbf { a } ^ { \prime }}$ & 0.75 & 0.77 \\
$\mathbf{4 \mathbf { b } ^ { \prime }}$ & 0.59 & 0.65 \\
$\mathbf{4 \mathbf { c } ^ { \prime }}$ & 0.51 & 0.44 \\
$\mathbf{4 d ^ { \prime }}$ & 0.97 & 1.74 \\
$\mathbf{4 \mathbf { e } ^ { \prime }}$ & 1.57 & 1.45 \\
$\mathbf{4 \mathbf { f } ^ { \prime }}$ & 0.47 & 0.78 \\
$\mathbf{4 \mathbf { g } ^ { \prime }}$ & 0.92 & 0.90 \\
\hline
\end{tabular}

\section{Materials and Methods}

\subsection{Chemistry}

All starting chemicals were commercially available and used as received, except that the solvents were purified by distillation.

IR spectra: PerkinElmer Spectrum One FT-IR spectrometer with a $\mathrm{KBr}$ disc, in $\mathrm{cm}^{-1}$; NMR spectra: Varian Gemini $200 \mathrm{MHz}$ spectrometer (200 MHz for ${ }^{1} \mathrm{H}$ and $50 \mathrm{MHz}$ for ${ }^{13} \mathrm{C}$ ), using $\mathrm{CDCl}_{3}$ as the solvent and TMS as the internal standard. ${ }^{1} \mathrm{H}$ and ${ }^{13} \mathrm{C}$ NMR chemical shifts were reported in parts per million (ppm) and were referenced to the solvent peak; $\mathrm{CDCl}_{3}\left(7.26 \mathrm{ppm}\right.$ for ${ }^{1} \mathrm{H}$ and 76.90 for ${ }^{13} \mathrm{C}$ ). Multiplicities are represented by s (singlet), $d$ (doublet), $t$ (triplet), $q$ (quartet), $d d$ (doublet of doublets), ddd (doublet of doublet of doublets) and $\mathrm{m}$ (multiplet). Coupling constants $(J)$ are in Hertz $(\mathrm{Hz})$. The melting point of products was determined by using MelTemp1000 apparatus.

\subsubsection{General procedure for synthesis of sulfonamides}

To a stirred solution of corresponding chalcone analogue $(1 \mathrm{mmol})$ in methanol $(25 \mathrm{ml})$ hydrazine hydrochloride ( $3 \mathrm{mmol}, 205 \mathrm{mg}$ ) and anhydrous $\mathrm{CH}_{3} \mathrm{COONa}(5 \mathrm{mmol}, 410 \mathrm{mg}$,) were added. The reaction mixture was heated to reflux overnight. The formed salt was filtered of and filtrate was cooled to room temperature. To stirred filtrate sulfonyl chloride $(1.5 \mathrm{mmol})$ was added. Then, triethylamine $(0.4 \mathrm{ml})$ was added dropwise and the resulting mixture was stirred overnight at room temperature. After completion of the reaction, mixture was poured into iced water and stirred well. The formed precipitate of sulfonamide product was filtered and dried in desiccator. 
5-(3-methoxy-4-propoxyphenyl)-3-methyl-1-(phenylsulfonyl)-4,5-dihydro-1H-pyrazole (3a'): Orange crystals; yield: 69\%; m.p. 107-108 ${ }^{\circ} \mathrm{C}$; IR (KBr): ): 3453, 2963, 2938, 2876, 1593, 1516, 1359, 1260, 1232, 1171, 1139, 1092, 1035, 725, $603 \mathrm{~cm}^{-1} ;{ }^{1} \mathrm{H}$ NMR (200 MHz, $\left.\mathrm{CDCl}_{3}\right): \delta=1.04\left(\mathrm{t}, 3 \mathrm{H}, J=7.6 \mathrm{~Hz}, \mathrm{CH}_{3}\right)$, $1.86\left(\mathrm{q}, 2 \mathrm{H}, J=7.4 \mathrm{~Hz}, \mathrm{CH}_{2}\right), 1.99\left(\mathrm{~s}, 3 \mathrm{H}, \mathrm{CH}_{3}\right), 2.70(\mathrm{ddd}, 1 \mathrm{H}, J=17.8,8.8,1.0 \mathrm{~Hz}, \mathrm{CH}), 3.06(\mathrm{ddd}, 1 \mathrm{H}, J$ $=17.6,11.0,0.6 \mathrm{~Hz}, \mathrm{CH}), 3.82\left(\mathrm{~s}, 3 \mathrm{H}, \mathrm{OCH}_{3}\right), 3.96\left(\mathrm{t}, 2 \mathrm{H}, J=6.8 \mathrm{~Hz}, \mathrm{OCH}_{2}\right), 4.79(\mathrm{dd}, 1 \mathrm{H}, J=11.0,8.8$ $\mathrm{Hz}, \mathrm{CH}), 6.81$ (s, 3H, Ar-H ), 7.44-7.59 (m, 3H, Ar-H), 7.79-7.83 (m, 2H, Ar-H); ${ }^{13} \mathrm{C}$ NMR $(50 \mathrm{MHz}$, $\left.\mathrm{CDCl}_{3}\right): \delta=10.4,15.9,22.5,47.6,56,64.2,70.6,110,112.8,119,128.4,128.6,132.9,133.2,136,148.3$, 149.6, 158.3 .

5-(3-methoxy-4-propoxyphenyl)-3-methyl-1-tosyl-4,5-dihydro-1H-pyrazole (3b'): Deep orange crystals; yield: $88 \%$; m.p. $120-121^{\circ} \mathrm{C}$; IR (KBr): 3438, 2963, 2939, 2876, 1596, 1516, 1359, 1260, 1231, 1167, 1138, 1093, 1035, 977, 673, $593 \mathrm{~cm}^{-1}$; ${ }^{1} \mathrm{H}$ NMR $\left(200 \mathrm{MHz}, \mathrm{CDCl}_{3}\right): \delta=1.04\left(\mathrm{t}, 3 \mathrm{H}, J=7.6 \mathrm{~Hz}, \mathrm{CH}_{3}\right)$, $1.86\left(\mathrm{q}, 2 \mathrm{H}, J=7.4 \mathrm{~Hz}, \mathrm{CH}_{2}\right), 1.99\left(\mathrm{~s}, 3 \mathrm{H}, \mathrm{CH}_{3}\right), 2.43\left(\mathrm{~s}, 3 \mathrm{H}, \mathrm{CH}_{3}\right), 2.69$ (ddd, $1 \mathrm{H}, J=18.0,9.0,0.8 \mathrm{~Hz}$, $\mathrm{CH}), 3.05(\mathrm{ddd}, 1 \mathrm{H}, J=17.4,11.0,0.6 \mathrm{~Hz}, \mathrm{CH}), 3.83\left(\mathrm{~s}, 3 \mathrm{H}, \mathrm{OCH}_{3}\right), 3.97\left(\mathrm{t}, 2 \mathrm{H}, J=6.8 \mathrm{~Hz}, \mathrm{OCH}_{2}\right), 4.75$ $(\mathrm{dd}, 1 \mathrm{H}, J=11.0,9.0 \mathrm{~Hz}, \mathrm{CH}), 6.81-6.84(\mathrm{~m}, 3 \mathrm{H}, \mathrm{Ar}-\mathrm{H}), 7.26-7.30(\mathrm{~m}, 2 \mathrm{H}, \mathrm{Ar}-\mathrm{H}), 7.70(\mathrm{dt}, 2 \mathrm{H}, J=8.2$, $1.8 \mathrm{~Hz}, \mathrm{Ar}-\mathrm{H}) ;{ }^{13} \mathrm{C} \mathrm{NMR}\left(50 \mathrm{MHz}, \mathrm{CDCl}_{3}\right): \delta=10.4,15.9,21.6,22.5,47.7,56,64.2,70.6,110.1,112.8,119$, $128.5,129.2,132.9,133.3,143.8,148.3,149.6,158.3$.

1-((4-(tert-butyl)phenyl)sulfonyl)-5-(3-methoxy-4-propoxyphenyl)-3-methyl-4,5-dihydro-1Hpyrazole (3c'): Orange crystals; yield: 87\%; m.p. 88-89 ${ }^{\circ} \mathrm{C}$; IR (KBr): 3454, 2964, 2875, 1595, 1516, 1359, 1263, 1232, 1171, 1140, 1088, 1036, 754, 633, $594 \mathrm{~cm}^{-1} ;{ }^{1} \mathrm{H}$ NMR $\left(200 \mathrm{MHz}, \mathrm{CDCl}_{3}\right): \delta=1.04(\mathrm{t}, 3 \mathrm{H}, J=7.4$ $\left.\mathrm{Hz}, \mathrm{CH}_{3}\right), 1.34\left(\mathrm{~s}, 9 \mathrm{H}, 3 \mathrm{CH}_{3}\right), 1.86\left(\mathrm{q}, 2 \mathrm{H}, J=7.2 \mathrm{~Hz}, \mathrm{CH}_{2}\right), 2.01\left(\mathrm{~s}, 3 \mathrm{H}, \mathrm{CH}_{3}\right), 2.71$ (ddd, $1 \mathrm{H}, J=18.0,9.0$, $1.0 \mathrm{~Hz}, \mathrm{CH}), 3.03-3.17(\mathrm{~m}, 1 \mathrm{H}, \mathrm{CH}), 3.81\left(\mathrm{~s}, 3 \mathrm{H}, \mathrm{OCH}_{3}\right), 3.96\left(\mathrm{t}, 2 \mathrm{H}, J=6.8 \mathrm{~Hz}, \mathrm{OCH}_{2}\right), 4.81(\mathrm{dd}, 1 \mathrm{H}, J=$ 11.0, $9.0 \mathrm{~Hz}, \mathrm{CH}), 6.76-6.84(\mathrm{~m}, 3 \mathrm{H}, \mathrm{Ar}-\mathrm{H}), 7.47(\mathrm{dt}, 2 \mathrm{H}, J=8.6,2.0 \mathrm{~Hz}, \mathrm{Ar}-\mathrm{H}), 7.72(\mathrm{dt}, 2 \mathrm{H}, J=8.8,2.0$ $\mathrm{Hz}, \mathrm{Ar}-\mathrm{H}) ;{ }^{13} \mathrm{C}$ NMR $\left(50 \mathrm{MHz}, \mathrm{CDCl}_{3}\right): \delta=10.5,15.9,22.5,31.1,35.2,47.7,56,64.2,70.6,110,112.8$, $119.1,125.6,127.8,128.3,133.3,148.3,149.6,156.7,158.1$.

5-(3-methoxy-4-propoxyphenyl)-1-((4-methoxyphenyl)sulfonyl)-3-methyl-4,5-dihydro-1H-

pyrazole (3d'): Light orange powder; yield: $85 \%$; m.p. 120-121 ${ }^{\circ} \mathrm{C}$; IR (KBr): 3437, 2962, 2939, 2876, $1594,1518,1359,1259,1235,1161,1135,1092,805,676.595 \mathrm{~cm}^{-1} ;{ }^{1} \mathrm{H}$ NMR $\left(200 \mathrm{MHz}, \mathrm{CDCl}_{3}\right): \delta=1.03$ $\left(\mathrm{t}, 3 \mathrm{H}, J=7.4 \mathrm{~Hz}, \mathrm{CH}_{3}\right), 1.86\left(\mathrm{q}, 2 \mathrm{H}, J=7.2 \mathrm{~Hz}, \mathrm{CH}_{2}\right), 1.99\left(\mathrm{~s}, 3 \mathrm{H}, \mathrm{CH}_{3}\right), 2.63-2.76(\mathrm{~m}, 1 \mathrm{H}, \mathrm{CH}), 2.98-3.13$ $(\mathrm{m}, 1 \mathrm{H}, \mathrm{CH}), 3.85(\mathrm{~d}, 6 \mathrm{H}, J=6.0 \mathrm{~Hz}, 2 \mathrm{OCH}), 3.96\left(\mathrm{t}, 2 \mathrm{H}, J=6.8 \mathrm{~Hz}, \mathrm{OCH}_{2}\right), 4.73(\mathrm{dd}, 1 \mathrm{H}, J=10.8,9.0$ $\mathrm{Hz}, \mathrm{CH}), 6.81-6.85(\mathrm{~m}, 3 \mathrm{H}, \mathrm{Ar}-\mathrm{H}), 6.95(\mathrm{dt}, 2 \mathrm{H}, J=8.8,2.0 \mathrm{~Hz}, \mathrm{Ar}-\mathrm{H}), 7.75(\mathrm{dt}, 2 \mathrm{H}, J=8.8,2.0 \mathrm{~Hz}$, $\mathrm{Ar}-\mathrm{H}) ;{ }^{13} \mathrm{C}$ NMR $\left(50 \mathrm{MHz}, \mathrm{CDCl}_{3}\right): \delta=10.4,15.9,22.5,47.7,55.6,56,64.3,70.6,110.1,112.8,113.8,119$, $127.4,130.6,133.4,148.2,149.5,158.3,163.2$.

1-((4-chlorophenyl)sulfonyl)-5-(3-methoxy-4-propoxyphenyl)-3-methyl-4,5-dihydro-1Hpyrazole (3e'): Yellow blonde powder; yield: 78\%; m.p. 159-160 ${ }^{\circ} \mathrm{C}$; IR (KBr): 3453, 2967, 2939, 2876, 1592, 1518, 1475, 1362, 1259, 1232, 1179, 1139, 1093, 1034, 759, $579 \mathrm{~cm}^{-1} ;{ }^{1} \mathrm{H}$ NMR $\left(200 \mathrm{MHz}, \mathrm{CDCl}_{3}\right): \delta$ $=1.04\left(\mathrm{t}, 3 \mathrm{H}, J=7.6 \mathrm{~Hz}, \mathrm{CH}_{3}\right), 1.87\left(\mathrm{q}, 2 \mathrm{H}, J=7.4 \mathrm{~Hz}, \mathrm{CH}_{2}\right), 2.01\left(\mathrm{~s}, 3 \mathrm{H}, \mathrm{CH}_{3}\right), 2.73(\mathrm{ddd}, 1 \mathrm{H}, J=17.8,8.4$, $1.0 \mathrm{~Hz}, \mathrm{CH}), 3.13(\mathrm{ddd}, 1 \mathrm{H}, J=17.8,11.0,0.8 \mathrm{~Hz}, \mathrm{CH}), 3.83\left(\mathrm{~s}, 3 \mathrm{H}, \mathrm{OCH}_{3}\right), 3.96(\mathrm{t}, 2 \mathrm{H}, J=6.8 \mathrm{~Hz}$, $\left.\mathrm{OCH}_{2}\right), 4.79(\mathrm{dd}, 1 \mathrm{H}, J=11.2,8.6 \mathrm{~Hz}, \mathrm{CH}), 6.76-6.79(\mathrm{~m}, 3 \mathrm{H}, \mathrm{Ar}-\mathrm{H}), 7.44(\mathrm{dt}, 2 \mathrm{H}, J=11.2,2.0 \mathrm{~Hz}$, Ar-H), $7.72(\mathrm{dt}, 2 \mathrm{H}, J=8.6,2.0 \mathrm{~Hz}, \mathrm{Ar}-\mathrm{H}) ;{ }^{13} \mathrm{C} \mathrm{NMR}\left(50 \mathrm{MHz}, \mathrm{CDCl}_{3}\right): \delta=10.5,15.9,22.5,47.6,56.1$, $64.3,70.6,110.2,112.8,119,128.8,129.7,132.7,134.8,139.5,148.5,149.5,158.5$.

1-((4-bromophenyl)sulfonyl)-5-(3-methoxy-4-propoxyphenyl)-3-methyl-4,5-dihydro-1H-pyrazole: Light amber powder; yield: 80\%; m.p. 155-156 ${ }^{\circ} \mathrm{C}$; IR (KBr): 3454, 2969, 2937, 2876, 1572, 1519, 1364, 1259, 1235, 1182, 1170, 1137, 1007, 751, $576 \mathrm{~cm}^{-1}$; ${ }^{1} \mathrm{H}$ NMR $\left(200 \mathrm{MHz}, \mathrm{CDCl}_{3}\right): \delta=1.04(\mathrm{t}, 3 \mathrm{H}, J=$ $\left.7.4 \mathrm{~Hz}, \mathrm{CH}_{3}\right), 1.87\left(\mathrm{q}, 2 \mathrm{H}, J=7.2 \mathrm{~Hz}, \mathrm{CH}_{2}\right), 2.01\left(\mathrm{~s}, 3 \mathrm{H}, \mathrm{CH}_{3}\right), 2.73(\mathrm{ddd}, 1 \mathrm{H}, J=17.8,8.4,0.8 \mathrm{~Hz}, \mathrm{CH}$ ), $3.13(\mathrm{ddd}, 1 \mathrm{H}, J=17.8,11.0,0.8 \mathrm{~Hz}, \mathrm{CH}), 3.82\left(\mathrm{~s}, 3 \mathrm{H}, \mathrm{OCH}_{3}\right), 3.96\left(\mathrm{t}, 2 \mathrm{H}, J=6.8 \mathrm{~Hz}, \mathrm{OCH}_{2}\right), 4.79(\mathrm{dd}$, $1 \mathrm{H}, J=11.0,8.4 \mathrm{~Hz}, \mathrm{CH}), 6.75-6.78(\mathrm{~m}, 3 \mathrm{H}, \mathrm{Ar}-\mathrm{H}), 7.56-7.67$ (m, 4H, Ar-H); ${ }^{13} \mathrm{C}$ NMR $(50 \mathrm{MHz}$, $\left.\mathrm{CDCl}_{3}\right): \delta=10.5,15.9,22.5,47.6,56.1,64.3,70.6,110.2,112.8,119,128,129.8,131.8,132.7,135.4,148.5$, $149.5,158.5$.

5-(3-methoxy-4-propoxyphenyl)-3-methyl-1-((4-nitrophenyl)sulfonyl)-4,5-dihydro-1H-pyrazole (3g'): Light amber powder; yield: 82\%; m.p. 150-151 ${ }^{\circ} \mathrm{C}$; IR (KBr): 3438, 2963, 2939, 2876, 1596, 1529, 1351, 1351, 1261, 1235, 1177, 1138, 1035, 750, $628 \mathrm{~cm}^{-1}$; ${ }^{1} \mathrm{H}$ NMR $\left(200 \mathrm{MHz}, \mathrm{CDCl}_{3}\right): \delta=1.05(\mathrm{t}$, $\left.3 \mathrm{H}, J=7.4 \mathrm{~Hz}, \mathrm{CH}_{3}\right), 1.87\left(\mathrm{q}, 2 \mathrm{H}, J=7.0 \mathrm{~Hz}, \mathrm{CH}_{2}\right), 2.05\left(\mathrm{~s}, 3 \mathrm{H}, \mathrm{CH}_{3}\right), 2.78(\mathrm{dd}, 1 \mathrm{H}, J=18.2,7.6 \mathrm{~Hz}, \mathrm{CH})$, 
$3.21(\mathrm{dd}, 1 \mathrm{H}, J=17.8,11.0 \mathrm{~Hz}, \mathrm{CH}), 3.81\left(\mathrm{~s}, 3 \mathrm{H}, \mathrm{OCH}_{3}\right), 3.94\left(\mathrm{t}, 2 \mathrm{H}, J=6.8 \mathrm{~Hz}, \mathrm{OCH}_{2}\right), 4.92(\mathrm{dd}, 1 \mathrm{H}, J=$ 11.2, 7.8 Hz, CH), 6.69-6.73 (m, 3H, Ar-H ), $7.89(\mathrm{~d}, 2 \mathrm{H}, J=8.6 \mathrm{~Hz}, \mathrm{Ar}-\mathrm{H}), 8.26(\mathrm{~d}, 2 \mathrm{H}, J=8.6 \mathrm{~Hz}$, Ar-H); ${ }^{13} \mathrm{C}$ NMR $\left(50 \mathrm{MHz}, \mathrm{CDCl}_{3}\right): \delta=10.4,16,22.5,47.3,56.1,64.3,70.6,110.4,112.6,119.1,123.6$, $129.2,132.1,142.6,148.8,149.5,150,158.8$.

5-(4-butoxy-3-methoxyphenyl)-3-methyl-1-(phenylsulfonyl)-4,5-dihydro-1H-pyrazole (4a'): Yellow orange crystals; yield: 70\%; m.p. 125-126 ${ }^{\circ}$; IR (KBr): 3432, 2957, 2933, 2871, 1593, 1517, 1361, 1259, 1229, 1166, 1137, 1033, 612, $567 \mathrm{~cm}^{-1} ;{ }^{1} \mathrm{H}$ NMR $\left(200 \mathrm{MHz}, \mathrm{CDCl}_{3}\right): \delta=0.98\left(\mathrm{t}, 3 \mathrm{H}, J=7.4 \mathrm{~Hz}, \mathrm{CH}_{3}\right)$, 1.40-1.59 (m, 2H, CH$), 1.75-1.89\left(\mathrm{~m}, 2 \mathrm{H}, \mathrm{CH}_{2}\right), 2.00\left(\mathrm{~s}, 3 \mathrm{H}, \mathrm{CH}_{3}\right), 2.64-2.77(\mathrm{~m}, 1 \mathrm{H}, \mathrm{CH}), 3.07(\mathrm{dd}, 1 \mathrm{H}, J$ $=18.0,11.0 \mathrm{~Hz}, \mathrm{CH}), 3.82\left(\mathrm{~s}, 3 \mathrm{H}, \mathrm{OCH}_{3}\right), 4.01\left(\mathrm{t}, 2 \mathrm{H}, J=6.6 \mathrm{~Hz}, \mathrm{OCH}_{2}\right), 4.79(\mathrm{dd}, 1 \mathrm{H}, J=11.2,8.8 \mathrm{~Hz}$, $\mathrm{CH}), 6.80$ (s, 3H, Ar-H ), 7.44-7.61 (m, 3H, Ar-H), 7.79-7.84 (m, 2H, Ar-H); ${ }^{13} \mathrm{C}$ NMR (50 MHz, CDCl 3 ): $\delta=13.9,15.9,19.2,31.2,47.6,56,64.3,68.8,109.9,112.7,119,128.4,128.6,132.9,133.1,135.9,148.3$, $149.5,158.4$.

5-(4-butoxy-3-methoxyphenyl)-3-methyl-1-tosyl-4,5-dihydro-1H-pyrazole (4b'): Deep orange crystals; yield: $90 \%$; m.p. 119-120 ${ }^{\circ} \mathrm{C}$; IR (KBr): 3432, 2958, 2934, 2872, 1596, 1516, 1360, 1259, 1231, 1167, 1138, 1033, 814, 673, $593 \mathrm{~cm}^{-1}$; ${ }^{1} \mathrm{H}$ NMR $\left(200 \mathrm{MHz}, \mathrm{CDCl}_{3}\right): \delta=0.98\left(\mathrm{t}, 3 \mathrm{H}, J=7.4 \mathrm{~Hz}, \mathrm{CH}_{3}\right)$, 1.40-1.59 (m, 2H, CH 2$), 1.75-1.90\left(\mathrm{~m}, 2 \mathrm{H}, \mathrm{CH}_{2}\right), 1.99\left(\mathrm{~s}, 3 \mathrm{H}, \mathrm{CH}_{3}\right), 2.43\left(\mathrm{~s}, 3 \mathrm{H}, \mathrm{CH}_{3}\right), 2.69(\mathrm{ddd}, 1 \mathrm{H}, J=$ $17.8,9.0,1.2 \mathrm{~Hz}, \mathrm{CH}), 3.05(\mathrm{ddd}, 1 \mathrm{H}, J=17.8,11.0,0.6 \mathrm{~Hz}, \mathrm{CH}), 3.83\left(\mathrm{~s}, 3 \mathrm{H}, \mathrm{OCH}_{3}\right), 4.01(\mathrm{t}, 2 \mathrm{H}, J=6.8$ $\left.\mathrm{Hz}, \mathrm{OCH}_{2}\right), 4.75(\mathrm{dd}, 1 \mathrm{H}, J=11.0,9.0 \mathrm{~Hz}, \mathrm{CH}), 6.81-6.83(\mathrm{~m}, 3 \mathrm{H}, \mathrm{Ar}-\mathrm{H}), 7.26-7.31(\mathrm{~m}, 2 \mathrm{H}, \mathrm{Ar}-\mathrm{H}), 7.71$ $(\mathrm{dt}, 2 \mathrm{H}, J=8.2,2.0 \mathrm{~Hz}, \mathrm{Ar}-\mathrm{H}) ;{ }^{13} \mathrm{C}$ NMR $\left(50 \mathrm{MHz}, \mathrm{CDCl}_{3}\right): \delta=13.9,16,19.3,21.7,31.3,47.7,56.1,64.3$, $68.8,110.1,112.8,119.1,128.6,129.3,133,133.4,143.9,148.4,149.6,158.4$.

5-(4-butoxy-3-methoxyphenyl)-1-((4-(tert-butyl)phenyl)sulfonyl)-3-methyl-4,5-dihydro-1Hpyrazole (4c'): Deep orange crystals; yield: $75 \%$; m.p. 81-82 ${ }^{\circ} \mathrm{C}$; IR (KBr): 3439, 2959, 2871, 1595, 1516, 1466, 1399, 1359, 1261, 1171, 1140, 1034, 754, 630, $594 \mathrm{~cm}^{-1} ;{ }^{1} \mathrm{H}$ NMR $\left(200 \mathrm{MHz}, \mathrm{CDCl}_{3}\right): \delta=0.98(\mathrm{t}, 3 \mathrm{H}$, $\left.J=7.2 \mathrm{~Hz}, \mathrm{CH}_{3}\right), 1.33\left(\mathrm{~s}, 9 \mathrm{H}, 3 \mathrm{CH}_{3}\right), 1.43-1.58\left(\mathrm{~m}, 2 \mathrm{H}, \mathrm{CH}_{2}\right), 1.76-1.86\left(\mathrm{~m}, 2 \mathrm{H}, \mathrm{CH}_{2}\right), 2.01\left(\mathrm{~s}, 3 \mathrm{H}, \mathrm{CH}_{3}\right)$, $2.71(\mathrm{ddd}, 1 \mathrm{H}, J=17.8,9.0,1.0 \mathrm{~Hz}, \mathrm{CH}), 3.10(\mathrm{ddd}, 1 \mathrm{H}, J=17.8,11.0,0.8 \mathrm{~Hz}, \mathrm{CH}), 3.83\left(\mathrm{~s}, 3 \mathrm{H}, \mathrm{OCH}_{3}\right)$, $4.00\left(\mathrm{t}, 2 \mathrm{H}, J=6.6 \mathrm{~Hz}, \mathrm{OCH}_{2}\right), 4.81(\mathrm{dd}, 1 \mathrm{H}, J=11.0,8.8 \mathrm{~Hz}, \mathrm{CH}), 6.81(\mathrm{~s}, 3 \mathrm{H}, \mathrm{Ar}-\mathrm{H}), 7.47(\mathrm{dt}, 2 \mathrm{H}, J=$ 8.6, $1.8 \mathrm{~Hz}, \mathrm{Ar}-\mathrm{H}), 7.72(\mathrm{dt}, 2 \mathrm{H}, J=8.6,1.8 \mathrm{~Hz}, \mathrm{Ar}-\mathrm{H}) ;{ }^{13} \mathrm{C} \mathrm{NMR}\left(50 \mathrm{MHz}, \mathrm{CDCl}_{3}\right): \delta=13.9,15.9,19.2$, 31.1, 31.3, 35.2, 47.7, 56, 64.2, 68.8, 110, 112.7, 119.1, 125.6, 127.8, 128.3, 133.2, 148.3, 149.5, 156.7, 158.1 .

5-(4-butoxy-3-methoxyphenyl)-1-((4-methoxyphenyl)sulfonyl)-3-methyl-4,5-dihydro-1H-pyrazole (4d'): Light orange powder; yield: 85\%; m.p. 88-89 ${ }^{\circ} \mathrm{C}$; IR (KBr): 3432, 2956, 2938, 2871, 1594, $1519,1357,1254,1157,1035,675,588 \mathrm{~cm}^{-1} ;{ }^{1} \mathrm{H} \mathrm{NMR}\left(200 \mathrm{MHz}, \mathrm{CDCl}_{3}\right): \delta=0.98\left(\mathrm{t}, 3 \mathrm{H}, J=7.4 \mathrm{~Hz}, \mathrm{CH}_{3}\right)$, 1.40-1.58 (m, 2H, CH 2$), 1.75-1.86\left(\mathrm{~m}, 2 \mathrm{H}, \mathrm{CH}_{2}\right), 1.99\left(\mathrm{~s}, 3 \mathrm{H}, \mathrm{CH}_{3}\right), 2.69(\mathrm{dd}, 1 \mathrm{H}, J=17.8,9.2 \mathrm{~Hz}, \mathrm{CH})$, $3.06(\mathrm{dd}, 1 \mathrm{H}, J=17.6,11.0, \mathrm{CH}), 3.85(\mathrm{~d}, 6 \mathrm{H}, J=7.2 \mathrm{~Hz}, 2 \mathrm{OCH}), 4.00\left(\mathrm{t}, 2 \mathrm{H}, J=6.8 \mathrm{~Hz}, \mathrm{OCH}_{2}\right), 4.74(\mathrm{dd}$, $1 \mathrm{H}, J=11.0,9.0 \mathrm{~Hz}, \mathrm{CH}), 6.81-6.84(\mathrm{~m}, 3 \mathrm{H}, \mathrm{Ar}-\mathrm{H}), 6.93-6.97(\mathrm{~m}, 2 \mathrm{H}, \mathrm{Ar}-\mathrm{H}), 7.73-7.78(\mathrm{~m}, 2 \mathrm{H}, \mathrm{Ar}-\mathrm{H})$; ${ }^{13} \mathrm{C}$ NMR $\left(50 \mathrm{MHz}, \mathrm{CDCl}_{3}\right): \delta=13.9,15.9,19.2,31.2,47.7,55.6,56,64.3,68.8,110,112.7,113.8,119$, $127.4,130.6,133.3,148.2,149.5,158.4,163.2$.

\section{5-(4-butoxy-3-methoxyphenyl)-1-((4-chlorophenyl)sulfonyl)-3-methyl-4,5-dihydro-1H-}

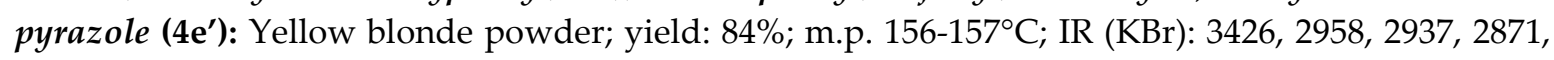
$1592,1518,1362,1258,1234,1179,1168,1137,1092,760,579 \mathrm{~cm}^{-1} ;{ }^{1} \mathrm{H} \mathrm{NMR}\left(200 \mathrm{MHz}, \mathrm{CDCl}_{3}\right): \delta=0.98$ $\left(\mathrm{t}, 3 \mathrm{H}, J=7.2 \mathrm{~Hz}, \mathrm{CH}_{3}\right), 1.41-1.59\left(\mathrm{~m}, 2 \mathrm{H}, \mathrm{CH}_{2}\right), 1.76-1.90\left(\mathrm{~m}, 2 \mathrm{H}, \mathrm{CH}_{2}\right), 2.02\left(\mathrm{~s}, 3 \mathrm{H}, \mathrm{CH}_{3}\right), 2.73(\mathrm{dd}, 1 \mathrm{H}$, $J=18.0,8.0 \mathrm{~Hz}, \mathrm{CH}), 3.13(\mathrm{dd}, 1 \mathrm{H}, J=17.8,11.2 \mathrm{~Hz}, \mathrm{CH}), 3.97\left(\mathrm{~s}, 3 \mathrm{H}, \mathrm{OCH}_{3}\right), 4.00(\mathrm{t}, 2 \mathrm{H}, J=6.8 \mathrm{~Hz}$, $\left.\mathrm{OCH}_{2}\right), 4.79(\mathrm{dd}, 1 \mathrm{H}, J=11.0,8.4 \mathrm{~Hz}, \mathrm{CH}), 6.76-6.79(\mathrm{~m}, 3 \mathrm{H}, \mathrm{Ar}-\mathrm{H}), 7.44(\mathrm{dt}, 2 \mathrm{H}, J=8.4,2.2 \mathrm{~Hz}, \mathrm{Ar}-\mathrm{H})$, $7.72(\mathrm{dt}, 2 \mathrm{H}, J=8.4,2.4 \mathrm{~Hz}, \mathrm{Ar}-\mathrm{H}) ;{ }^{13} \mathrm{C} \mathrm{NMR}\left(50 \mathrm{MHz}, \mathrm{CDCl}_{3}\right): \delta=13.9,16,19.3,31.3,47.6,56.1,64.4$, $68.9,110.3,112.8,119.1,128.9,129.8,132.8,134.9,139.5,148.6,149.6,158.6$.

1-((4-bromophenyl)sulfonyl)-5-(4-butoxy-3-methoxyphenyl)-3-methyl-4,5-dihydro-1H-pyrazole (4f'): Yellow orange powder; yield: 75\%; m.p. 136-137 ${ }^{\circ} \mathrm{C}$; IR (KBr): 3426, 2958, 2956, 2872, 1575, $1518,1362,1258,1233,1170,1137,1010,751,575 \mathrm{~cm}^{-1} ;{ }^{1} \mathrm{H}$ NMR $\left(200 \mathrm{MHz}, \mathrm{CDCl}_{3}\right): \delta=0.99(\mathrm{t}, 3 \mathrm{H}, J=$ $\left.7.2 \mathrm{~Hz}, \mathrm{CH}_{3}\right), 1.41-1.59\left(\mathrm{~m}, 2 \mathrm{H}, \mathrm{CH}_{2}\right), 1.76-1.90\left(\mathrm{~m}, 2 \mathrm{H}, \mathrm{CH}_{2}\right), 2.02\left(\mathrm{~s}, 3 \mathrm{H}, \mathrm{CH}_{3}\right), 2.74(\mathrm{ddd}, 1 \mathrm{H}, J=17.8$, 8.2, $0.6 \mathrm{~Hz}, \mathrm{CH}), 3.13(\mathrm{ddd}, 1 \mathrm{H}, J=17.8,11.0,0.4 \mathrm{~Hz}, \mathrm{CH}), 3.83\left(\mathrm{~s}, 3 \mathrm{H}, \mathrm{OCH}_{3}\right), 4.01(\mathrm{t}, 2 \mathrm{H}, J=6.6 \mathrm{~Hz}$, $\left.\mathrm{OCH}_{2}\right), 4.80(\mathrm{dd}, 1 \mathrm{H}, J=11.0,8.4 \mathrm{~Hz}, \mathrm{CH}), 6.75-6.78(\mathrm{~m}, 3 \mathrm{H}, \mathrm{Ar}-\mathrm{H}), 7.57-7.67(\mathrm{~m}, 4 \mathrm{H}, \mathrm{Ar}-\mathrm{H}) ;{ }^{13} \mathrm{C} \mathrm{NMR}$ (50 MHz, $\left.\mathrm{CDCl}_{3}\right): \delta=13.8,15.9,19.2,31.2,47.5,56,64.3,68.7,110.1,112.6,118.9,128,129.7,131.8$, 132.6, 135.3, 148.4, 149.5, 158.5. 
5-(4-butoxy-3-methoxyphenyl)-3-methyl-1-((4-nitrophenyl)sulfonyl)-4,5-dihydro-1H-pyrazole (4g'): Yellow blonde powder; yield: 82\%; m.p. 138-139 ${ }^{\circ} \mathrm{C}$; IR (KBr): 3432, 2961, 2943, 2872, 1594, 1529, $1367,1360,1347,1259,1174,1136,1034,741,626,578 \mathrm{~cm}^{-1} ;{ }^{1} \mathrm{H}$ NMR $\left(200 \mathrm{MHz}, \mathrm{CDCl}_{3}\right): \delta=0.99(\mathrm{t}, 3 \mathrm{H}$, $\left.J=7.4 \mathrm{~Hz}, \mathrm{CH}_{3}\right), 1.41-1.60\left(\mathrm{~m}, 2 \mathrm{H}, \mathrm{CH}_{2}\right), 1.76-1.90\left(\mathrm{~m}, 2 \mathrm{H}, \mathrm{CH}_{2}\right), 2.05\left(\mathrm{~s}, 3 \mathrm{H}, \mathrm{CH}_{3}\right), 2.78$ (ddd, $1 \mathrm{H}, J=$ $17.8,7.8,0.6 \mathrm{~Hz}, \mathrm{CH}), 3.21(\mathrm{ddd}, 1 \mathrm{H}, J=17.8,11.0,0.6 \mathrm{~Hz}, \mathrm{CH}), 3.81\left(\mathrm{~s}, 3 \mathrm{H}, \mathrm{OCH}_{3}\right), 3.98(\mathrm{t}, 2 \mathrm{H}, J=6.8$ $\left.\mathrm{Hz}, \mathrm{CH}_{2}\right), 4.92(\mathrm{dd}, 1 \mathrm{H}, J=11.0,7.4 \mathrm{~Hz}, \mathrm{CH}), 6.63-6.74(\mathrm{~m}, 3 \mathrm{H}, \mathrm{Ar}-\mathrm{H}), 7.89(\mathrm{dt}, 2 \mathrm{H}, J=8.6,2.2 \mathrm{~Hz}$, Ar-H), 8.26 (dt, $2 \mathrm{H}, J=8.4,2.2 \mathrm{~Hz}, \mathrm{Ar}-\mathrm{H}) ;{ }^{13} \mathrm{C}$ NMR $\left(50 \mathrm{MHz}, \mathrm{CDCl}_{3}\right): \delta=13.9,16.1,19.3,31.3,47.4$, $56.1,64.4,68.9,110.4,112.6,119.1,123.7,129.3,132.1,142.6,148.9,149.5,150.1,158.9$.

\subsection{Cytotoxicity}

Cytotoxicity screening of fourteen organic compounds was performed on two human tumor cell lines, cervix adenocarcinoma (HeLa) and colorectal adenocarcinoma (SW480), and non-transformed human lung fibroblast cells (MRC-5). All three cell lines were obtained from American Type Culture Collection (ATTC). Cells were grown as a monolayer in Dulbecco's modified Eagle's medium (DMEM) supplemented with 10\% heath-inactivated foetal bovine serum (FBS), L-glutamine $(2 \mathrm{mM})$, non-essential amino acids $(0,1 \mathrm{mM})$, penicillin $(100 \mathrm{IU} / \mathrm{ml})$ and streptomycin $(100 \mu \mathrm{g} / \mathrm{ml})$ under standard culture conditions of $37^{\circ} \mathrm{C}$, humidified air and $5 \% \mathrm{CO}_{2}$. The cell cultures were subpassaged at $80 \%$ of confluency, seeded in 96 -well microtitar plates at the density of $3 \times 10^{3}$ cells per well and incubated to adhere overnight. In vitro cytotoxicity was evaluated using MTT colorimetric assay. Plated cells were treated with fourteen organic compounds in a single concentration $100 \mu \mathrm{M}$ or media alone, as control, during 48h. After treatment period, MTT solution $(0.5 \mathrm{mg} / \mathrm{ml}$ in supplemented medium) was added to each well for at least $2 \mathrm{~h}$. Subsequently, supernatant was removed and formazan crystals were dissolved with $150 \mu \mathrm{l}$ of DMSO. Absorbance was measured at $590 \mathrm{~nm}$ with a multiplate reader (Zenyth 3100, Anthos Labtec Instruments $\mathrm{GmbH}$, Austria). All experiments were repeated at least three times (six wells per experiment). Cytotoxicity was calculated according to the formula: (1-A test/A control) $\times 100$. The cytotoxicity ratio (CR) was calculated as ratio between $\%$ of cytotoxicity of each compound on normal MRC- 5 cell line and \% of cytotoxicity of the same compounds on SW480 and Hela tumor cell line. Compounds with CR value lower than 1 were considered selective to tumor cells over normal cells.

\section{Conclusion}

Due to our research interest for the synthesis of molecules exhibiting some biological activity, we decided to synthesize a new sulfonamide compounds which have vanillin fragment and pyrazoline ring in their structure. In this study sulfonamide compounds of dehydrozingerone derivatives were prepared in very good yields and characterized by their spectral data. Results of cytotoxicity screening showed that some compounds could be good candidates for further investigation. Having in mind selective nature of some butyl derivatives (4 $\mathbf{4} \mathbf{b}^{\prime}$ and $\mathbf{4} \mathbf{f}^{\prime}$ ) against SW480 and Hela tumor cells, the mechanism of observed cytotoxic activity should be further evaluated in terms of elucidating type of cell death and cell cycle perturbation.

\section{Author Contributions:}

“Conceptualization, Zoran Ratković, Jovana Muškinja, Suzana Popović and Danijela Todorović; methodology, Zoran Ratković, Jovana Muškinja and Suzana Popović; software, Sanja Matić.; validation, Jovana Muškinja, Suzana Popović, Danijela Todorović and Dejan Baskić; formal analysis, Zoran Ratković, Jovana Muškinja and Sanja Matić; investigation, Zoran Ratković, Jovana Muškinja, Sanja Matić, and Suzana Popović; resources, Zoran Ratković, Jovana Muškinja, Dejan Baskić and Danijela Todorović; data curation, Sanja Matic and Suzana Popović.; writing - original draft preparation, Jovana Muškinja and Sanja Matić; writing - review and editing, Zoran Ratković and Suzana Popović; visualization, Jovana Muškinja, Sanja Matić and Suzana Popović; supervision, Zoran Ratković and Dejan Baskić; project administration, Zoran Ratković, Dejan Baskić and Danijela Todorović.; funding acquisition, Zoran Ratković, Dejan Baskić and Danijela Todorović."

Funding: "This research received no external funding" 
Acknowledgments: The authors are grateful to the Ministry of Education, Science and Technological Development of the Republic of Serbia (Grant 172034) for financial support.

Conflicts of Interest: “The authors declare no conflict of interest.”

\section{References}

1. Motohashi, N.; Yamagami, C.; Tokuda, H.; Konoshima, T.; Okuda, Y.; Okuda, M.; Mukainaka, T.; Nishino, H.; Saito, Y. Inhibitory effects of dehydrozingerone and related compounds on 12-O-tetradecanoylphorbol-13-acetate induced Epstein-Barr virus early antigen activation. Cancer Lett. 1998, 134, 37-42.

2. Adams, B.K.; Ferstl, E.M.; Davis, M.C.; Herold, M.; Kurtlaya, S.; Camalier, R.F.; Hollingshead, M.G.; Kaur, G.; Sausville, E.A.; Rickles, F.R.; Snyder, J.P.; Liotta, D.C.; Shoji, M. Synthesis and biological evaluation of novel curcumin analogs as anti-cancer and anti-angiogenesis agents. Bioorg. Med. Chem. 2004, 12, 3871-3883.

3. Ishida, J.; Ohtsu, H.; Tachibana, Y.; Nakanishi, Y.; Bastow, K.F.; Nagai, M.; Wang, H.K.; Itokawa, H.; Lee, K.H. Antitumor agents. Part 214: synthesis and evaluation of curcumin analogues as cytotoxic agents. Bioorg. Med. Chem. 2002, 10, 3481-3487.

4. Kalirajan, R.; Sivakumar S.U.; Jubie, S.; Gowramma, B., Suresh, B. Synthesis and biological evaluation of some heterocyclic derivatives of chalcones. Int. J. ChemTech. Res. 2009, 1, $27-34$.

5. Varga, L.; Nagy, T.; Kövesdi, I.; Benet-Bucholz, J.; Dorman, G.; Urge, L.; Darvas, F. Solution-phase parallel synthesis of 4,6-diaryl-pyrimidine-2-ylamines and 2-amino-5,5-disubstituted-3,5-dihydro-imidazol-4-ones via a rearrangement. Tetrahedron 2003, 59, 655-662.

6. Burmudžija, A.; Muškinja, J.; Ratković, Z.; Kosanić, M.; Ranković, B.; Novaković, S.; Bogdanović, G. Pyrazoline derivatives of acryloyl substituted ferrocenyl ketones: synthesis, antimicrobial activity and structural properties. Inorg. Chim. Acta, 2018, 471, 570-576.

7. Levai, A.J. Synthesis of 2-pyrazolines by the reactions of $\alpha, \beta$-unsaturated aldehydes, ketones, and esters with diazoalkanes, nitrile, imines, and hydrazines. J. Heterocycl. Chem. 2002, 39, 1-13.

8. Kissane, M.; Maguire, A.R. Asymmetric 1,3-dipolar cycloadditions of acrylamides. Chem. Soc. Rev. 2010, 39, 845-883.

9. Ali, M.A.; Shaharyar, M. Discovery of novel phenoxyacetic acid derivatives as antimycobacterial agents. Bioorg. Med. Chem. 2007, 15, 1896-1902.

10. Sahoo, A.; Yabanoglu, S.; Sinha, B.N.; Ucar, G.; Basu, A.; Jayaprakash, V. Towards development of selective and reversible pyrazoline based MAO-inhibitors: Synthesis, biological evaluation and docking studies. Bioorg. Med. Chem. Lett. 2010, 20, 132-136.

11. Casini, A.; Scozzafava, A.; Mastrolorenzo, A.; Supuran, C.T. Sulfonamides and sulfonylated derivatives as anticancer agents. Curr Cancer Drug Targets, 2002, 2, 55-75.

12. Fares, M, Eladwy, RA, Nocentini, A, El Hadi, S.R.A.; Ghabbour, H.A.; Abdel-Megeed, A.; Eldehna, W.M.; Abdel-Aziz, H.A.; Supuran, C.T. Synthesis of bulky-tailed sulfonamides incorporating pyrido [2, 3-d][1, 2, 4]-triazolo-[4, 3-a]-pyrimidin-1-(5H)-yl) moieties and evaluation of their carbonic anhydrases I, II, IV and IX inhibitory effects. Bioorg Med Chem. 2017, 25, 2210-2217.

13. Neu, H.C.; Gootz, T.D. Medical Microbiology, Baron, S. Ed., The University of Texas Medical Branch at Galveston, Galveston 1996.

14. Owa, T.; Nagasu, T. Novel sulphonamide derivatives for the treatment of cancer. Exp. Opin. Ther. Pat. 2000, 10, 1725-1740.

15. Ozawa, Y.; Sugi1, N.H.; Nagasu, T.; Owa, T.; Watanabe, T.; Koyanagi, N.; Yoshino, H.; Kitoh, K.; Yoshimatsu, K. E7070, a novel sulphonamide agent with potent antitumor activity in vitro and in vivo. Eur. J. Cancer 2001, 37, 2275-2282.

16. Ratković, Z.; Muškinja, J.; Burmudžija, A.; Ranković, B.; Kosanić, M.; Bogdanović, G.A.; Marković, B.S.; Nikolić, A.; Arsenijević, N.; Đorđević, S.; Vukićević, R.D. Dehydrozingerone based 1-acetyl-5-aryl-4,5-dihydro-1H-pyrazoles: Synthesis, characterization and anticancer activity. J. Mol. Struc. 2016, 1109, 82-88.

17. Muškinja, J.; Ratković, Z.; Ranković, B.; Kosanić, M. Synthesis of O-alkyl derivatives of dehydrozingerone analogues. Kragujevac J. Sci. 2016, 38, 97-106. 